\title{
Anforderung an die Energieversorgung von Großkrankenhäusern
}

\author{
M. Zaufl, H. Hollaus \\ Online publiziert am 20. November 2017 \\ (c) Springer-Verlag GmbH Austria, ein Teil von Springer Nature 2017
}

Die besonderen Anforderungen an die Energieversorgung ergeben sich vor allem aus der Größe und den Aufgaben des Krankenhauses. Am Beispiel „Allgemeines Krankenhaus der Stadt Wien - Medizinischer Universitätscampus" (AKH Wien) werden die besonderen Anforderungen erläutert.

In dem seit 1991 in Betrieb befindlichen größten Spital Österreichs mit $900.000 \mathrm{~m}^{2}$ Nettogrundrissfläche befinden sich die klinischen Bereiche, die Einrichtungen der Medizinischen Universität, Forschungsstätten und Ausbildungszentren. 10.000 Mitarbeiter sorgen für medizinische Spitzenleistungen an den 26 Universitätskliniken und an 42 Klinischen Abteilungen und Instituten. Das Studienzentrum bietet Platz für rd. 3.000 Studierende. Für den reibungsIosen technischen Betrieb und die Instandhaltung der haustechnischen und medizinischen Einrichtungen ist die VAMED-KMB zuständig.

Der Medienverbrauch für den elektrischen Strom beträgt jährlich 155.000 MWh, Erdgas 36.000 MWh und Fernwärme 182.000 MWh. Der Wasserbedarf liegt bei jährlich $866.000 \mathrm{~m}^{3}$. Das für die Klimatisierung der 27.000 Räume und die Kühlung der technischen Anlagen benötigte Kaltwasser von $5,5^{\circ} \mathrm{C}$ wird mit Turbo- und AbsorberKältemaschinen erzeugt. Rund die Hälfte des Kältebedarfs von jährlich 80.000 MWh wird durch Fernkälte der „Wien Energie Fernwärme" geliefert. Alle technischen Anlagen werden über eine zentrale Leittechnik mit 78.000 Datenpunkten überwacht bzw. gesteuert.

Die elektrischen Anlagen und Installationen des AKH Wien werden über 95 Gießharztransformatoren $10 \mathrm{kV} / 400 \mathrm{~V}$, aufgeteilt auf 16 Trafostationen, versorgt. 3.500 Elektroverteiler speisen ca. 80.000 Leuchten sowie rd. 180.000 Steckdosen und Geräteauslässe.

Zwei Regelumspanner 110 kV/10 kV mit je 40 MW im benachbarten Umspannwerk versorgen das AKH vollredundant. Die Notstromversorgung ist über zentrale Dieselaggregate mit einer um 10\% überlastbaren Gesamtleistung von $18.800 \mathrm{~kW}$ sichergestellt. Statische Batterieanlagen und rotierende USV-Anlagen mit jeweils bis zu 700 kW sind für den Betrieb der Operationsräume, Intensivstationen, angiografischen Anlagen, Rechenzentren, Forschungseinrichtungen etc. notwendig. Der Bedarf an USV-gestützter Stromversorgung nimmt stetig zu.

Die Überwachung der elektrotechnischen Anlagen erfolgt rund um die Uhr in der Leitwarte des AKH Wien über das Leitsystem SINAUT Spectrum. Dieses hat in einem Erneuerungsschritt 2006 bis 2011 den um 1988 errichteten Prozessrechner samt zugehöriger Fernwirksysteme FW535 und FW537 abgelöst.

Die technische Klärung von gleichzeitig auftretenden Störungsmeldungen an Anlagen des AKH Wien - vor allem bei externen Netzeinsenkungen - erfolgt durch den unmittelbaren Zugriff auf die Stördatenerfassung.
In den Trafostationen sind netzwerkverbundene Stationsleitsysteme SICAM PAS sowie SIPROTEC als Feldsteuer- und Schutzgeräte eingesetzt. Wie bei fast allen Anlagenerneuerungen konnten auch diese Umsetzungen nur step by step durchgeführt werden. Eine auch nur kurzzeitige Verringerung der Versorgungssicherheit konnte wegen der Zentralfunktion nicht akzeptiert werden.

Weiters wurde 2016 mit der Erneuerung aller SPS im Bereich der Dieselsteuerungen, der übergeordneten Notnetzautomatiken und zentralen Synchronisierungs-Steuerungen begonnen. Das gesamte Equipment ist nun redundant ausgeführt, die Betriebs- und Versorgungssicherheit damit wesentlich gesteigert. Die nächsten Modernisierungsschritte sehen ab 2020 die Erneuerung des Leitsystems mit Portierung der 20.000 projektspezifischen Erweiterungen sowie die Berücksichtigung der Cybersecurity-Vorgaben vor.

Auf Verlangen von E-Control muss seit 2015 bei Umschaltung der aktiven Anspeisung auf die redundante 10-kV-Anspeisung im betreffenden Monat für beide Anspeisungen das Nutzungsentgelt verrechnet werden. Davor wurden beide Anspeisungen virtuell zu einem Zählpunkt zusammengefasst. Zur Vermeidung der monatlichen Mehrkosten von rd. 96.000,- EUR müssen nun alle Instandhaltungsmaßnahmen im Umspannwerk und im AKH zeitlich komprimiert werden. Zwischenzeitliche Funktionstests sind kostenintensiv.

Bei der Anschaffung von Großgeräten ist die Verbrauchszählung dieser Geräte samt Hilfsanlagen technischer Standard. Die Aufbereitung dieser Daten ist Teil des umfangreichen Energiemanagements.

Erhöhte Anforderungen an die Netzqualität bestehen bei der Versorgung der Rechenzentren. Mit der Qualitätsstufe "Tier 4" ist u. a. eine Verfügbarkeit von 99,991\% verbunden. Neben voneinander unabhängigen Versorgungswegen und rasch verfügbaren Ersatzaggregaten erfolgt bereits beim Auftreten geringer Ableitströme an den Servern eine Vorwarnung.

Besondere Anforderungen bestehen in Bezug auf ein mögliches „Blackout”, dem längerfristigen Ausfall der öffentlichen Stromversorgung. Ohne Neuversorgung mit dem notwendigen Dieselkraftstoff endet der Notstrombetrieb bereits am zweiten Tag, eine Leistungsreduktion kann die Versorgungszeit auf einige Tage erweitern.

Besondere Herausforderungen sind die künftigen, bereits beschlossenen Umbauten, baulichen Sanierungen, Modernisierungen div. Kliniken und die Errichtung mehrerer medizinischer Zentren bis zum Jahr 2030 am Gelände des AKH Wien.

Kurzfassung eines Vortrags bei der 55. Fachtagung der Österreichischen Gesellschaft für Energietechnik (OGE) im OVE, die am 12. und 13. Oktober 2017 in Salzburg stattfand.

Zaufl, Michael, VAMED-KMB Krankenhausmanagement und Betriebsführungsges. m.b.H., Spitalgasse 23, 1090 Wien, Österreich (E-Mail: michael.zaufl@vamed.com); Hollaus, Herbert, VAMED-KMB Krankenhausmanagement und Betriebsführungsges. m.b.H., Spitalgasse 23, 1090 Wien, Österreich 